\title{
Slit2 Guides Both Precrossing and Postcrossing Callosal Axons at the Midline In Vivo
}

\author{
Tianzhi Shu, ${ }^{1}$ Vasi Sundaresan, ${ }^{4}$ Margaret M. McCarthy, ${ }^{2,3}$ and Linda J. Richards ${ }^{1,3}$ \\ Departments of ${ }^{1}$ Anatomy and Neurobiology and ${ }^{2}$ Physiology and ${ }^{3}$ Program in Neuroscience, The University of Maryland, Baltimore, School of Medicine, \\ Baltimore, Maryland 21201, and “Department of Histopathology, St. Thomas' Hospital, London SE1 7EH, United Kingdom
}

Commissural axons generally cross the midline only once. In the Drosophila nerve cord and mouse spinal cord, commissural axons are guided by Slit only after they cross the midline, where Slit prevents these axons from recrossing the midline. In the developing corpus callosum, Slit2 expressed by the glial wedge guides callosal axons before they cross the midline, as they approach the corticoseptal boundary. These data highlighted a potential difference between the role of Slit 2 in guiding commissural axons in the brain compared with the spinal cord. Here, we investigate whether Slit2 also guides callosal axons after they cross the midline. Because such questions cannot be addressed in conventional gene knock-out animals, we used in utero injections of antisense oligonucleotides to specifically deplete Slit2 on only one side of the brain. We used this technique together with a novel in vitro assay of hemisected brain slices to specifically analyze postcrossing callosal axons. We find that in the brain, unlike the spinal cord, Slit 2 mediates both precrossing and postcrossing axonal guidance. Depletion of Slit 2 on one side of the brain causes axons to defasciculate and, in some cases, to aberrantly enter the septum. Because these axons do not recross the midline, we conclude that the principle function of Slit 2 at the cortical midline may be to channel the axons along the correct path and possibly repel them away from the midline. We find no evidence that Slit 2 prevents axons from recrossing the midline in the brain.

Key words: corpus callosum; commissure; axon guidance; cortical development; glial wedge; midline; Slit; Robo; antisense oligonucleotides

\section{Introduction}

Commissural axons comprising the corpus callosum connect neurons in the right and left cortical hemispheres. During development, callosal axons arising principally from neurons in layers $2 / 3$ and 5 project initially to the intermediate zone, probably under the influence of class 3 semaphorins (Polleux et al., 1998). Callosal axons grow medially and ventrally to reach the corticoseptal boundary, at which they encounter a bilaterally symmetrical structure called the glial wedge (Shu and Richards, 2001), and turn sharply to cross the midline. After crossing the midline, callosal axons encounter the contralateral glial wedge and make another turn dorsally to enter the intermediate zone of the contralateral cingulate cortex. These striking morphological changes in the direction of callosal axon trajectories in vivo suggest that these axons are being guided by molecules within the local environment. Additional evidence of this is the large number of genetic mutations that result in agenesis of the corpus callosum (for review, see Richards, 2002) and cause a similar acallosal phenotype. In most cases, axons are able to reach the midline but are

\footnotetext{
Received March 12, 2003; revised July 18, 2003; accepted July 21, 2003.

This work was supported by National Institutes of Health-National Institute of Neurological Disorders and Stroke Grant NS37792 (L.J.R.). V.S. is a recipient of a Medical Research Council (United Kingdom) Career Establishment Award. We gratefully acknowledge the technical contributions of Kimberly M. Valentino, Aika S. Mongi, and Adelaide Annan. We thank Drs. M. Tessier-Lavigne and E. Stein for the gifts of Robo ectodomain expression plasmids. Correspondence should be addressed to Dr. Linda J. Richards, Department of Anatomy and Neurobiology, The University of Maryland, Baltimore, HSF 222, 685 West Baltimore Street, Baltimore, MD 21201. E-mail: Irich001@umaryland.edu.

Copyright $\odot 2003$ Society for Neuroscience $\quad 0270-6474 / 03 / 238176-09 \$ 15.00 / 0$
}

unable to cross, continuously growing into large swirls of axons known as Probst bundles. This phenotype is apparent whether the gene mutation affects callosal axons intrinsically (Shen et al., 2002) or whether the mutation effects the development of midline structures (Shu et al., 2003) or the molecules they express (Bagri et al., 2002).

Midline glial structures have been shown to regulate commissural axon guidance in a number of systems (Van Hartesveldt et al., 1986; Jacobs and Goodman, 1989; Mori et al., 1990; Snow et al., 1990; Marcus et al., 1995; Cummings et al., 1997; Pires-Neto et al., 1998; Wu et al., 1998). Work in invertebrates and in the vertebrate spinal cord has begun to elucidate some of the molecules involved in midline glial directed guidance. One of these molecules, Slit2, and its Robo receptors were first identified in Drosophila and then subsequently cloned in mammals (Kidd et al., 1998, 1999; Brose et al., 1999; Li et al., 1999; Wang et al., 1999). In Drosophila, and at the rodent cortical midline, Slit2 is expressed by midline glia (Kidd et al., 1999; Nuygen-Ba Charvet et al., 1999; Shu and Richards, 2001). In Slit2 knock-out mice, callosal axons form Probst bundles, and laterally projecting axons form ectopic commissures and project aberrantly into ventral regions of the basal telencephalon (Bagri et al., 2002). These results suggest a role for Slit2 in guiding cortical axons as they approach the midline before they cross.

In vitro "open book" assays of the developing spinal cord show that Slit2 and class 3 semaphorins regulate commissural axon guidance only after the axons cross the midline (Zou et al., 2000), 
A

$220 \mathrm{kDa}$

$97 \mathrm{kDa}$

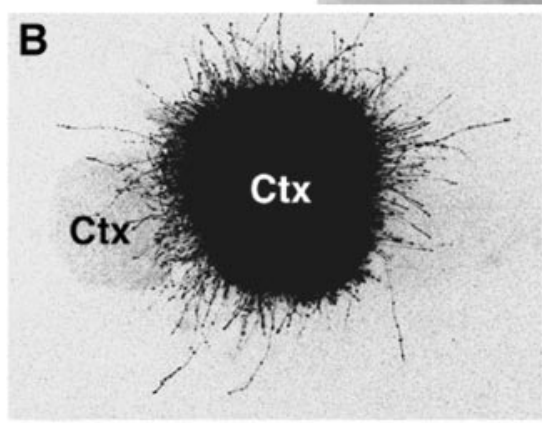

D

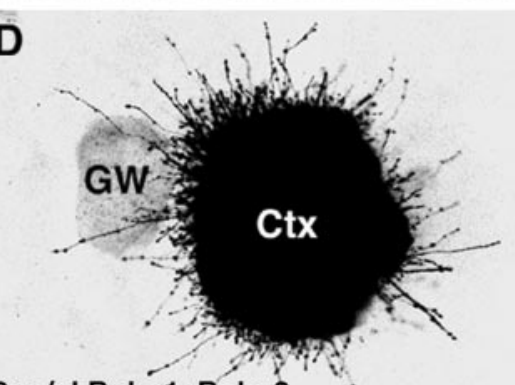

2ng/ul Robo1+Robo2

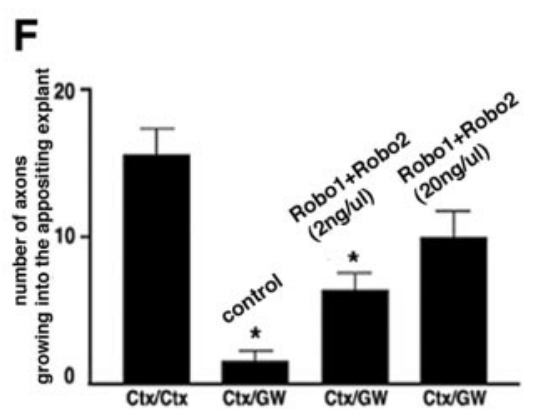

C
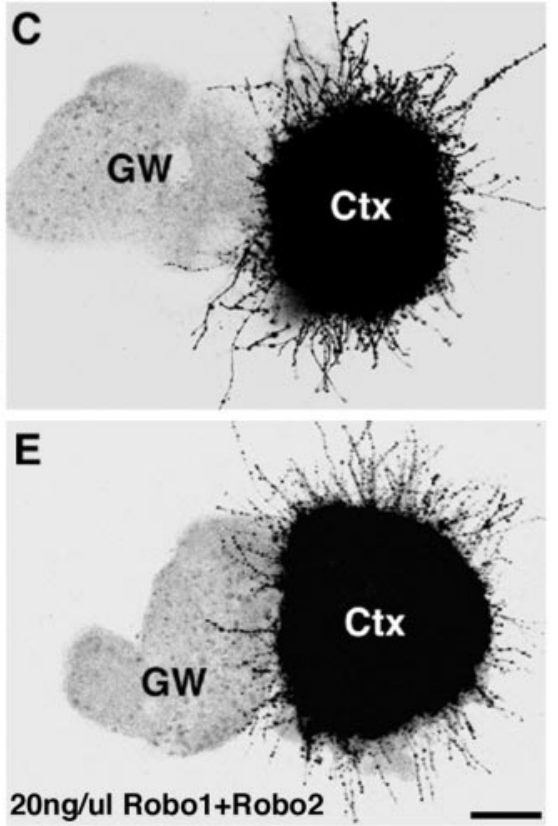

\section{G}

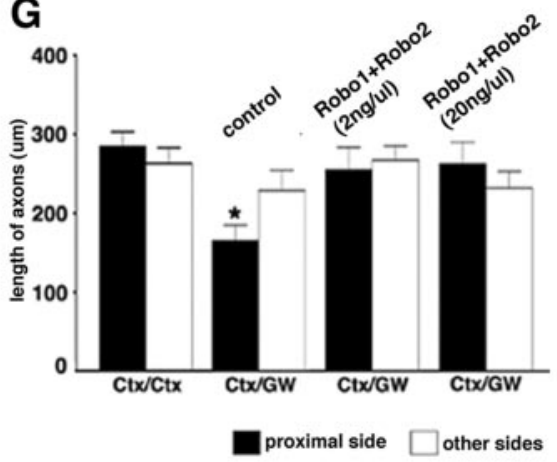

Figure 1. Robo ectodomain proteins block the chemosuppressive-repulsive effect of the glial wedge. Robo1 and Robo2 ectodomain proteins were synthesized using an in vitro transcription-translation system and visualized by Western blot analysis by the incorporation of biotinylated lysines $(A)$. Both Robo1 and Robo2 ectodomain proteins were produced with an estimated mass of 150-160 kDa (A, lanes 2 and 3), consistent with previous findings (Brose et al., 1999). In the control lane (A, lane 1), an empty vector was added to the reaction, and, thus, no protein was produced. Collagen gel experiments were performed using explants derived from E17 brain slices. Cortical explants (Ctx) were cocultured with glial wedge $(\mathrm{GW})$ explants $(C)$ or another cortical explant $(B)$ as controls. As described previously, the $G W$ causes a growth suppression-repulsion of cortical axons $(B)$. Robo ectodomain proteins were mixed and added to the growth medium of Ctx/GW cocultures at either $2 \mathrm{ng} / \mu$ l total protein (D) or 20 $\mathrm{ng} / \mu$ l total protein $(E)$. After $3 \mathrm{~d}$ in culture, explants were fixed, and Dil was injected into the cortical explant to label the axons. $F, G$, Significantly more, and longer, axons grew into the GW when either $2 \mathrm{ng} / \mu \mathrm{l}$ or $20 \mathrm{ng} / \mu \mathrm{l}$ of the Robo ectodomain proteins was added to the cultures $\left({ }^{*} p<0.05\right.$; ANOVA), indicating the inhibitory effect of the $\mathrm{GW}$ had been blocked. Histograms represent the mean \pm SEM. Scale bar (in $E): B-E, 150 \mu \mathrm{m}$.

although this has not been demonstrated in vivo. Here, we investigate whether in the brain Slit2 regulates axonal guidance both before and after callosal axons cross the midline. These results demonstrate a significant difference between how this molecule regulates the development of commissures in the spinal cord and brain.

\section{Materials and Methods}

Animals and perfusion. Embryos used in this study were obtained from timed pregnant C57BL/6J mice (Jackson Laboratories, Bar Harbor, ME). Females were checked for the presence of a vaginal plug after overnight mating with a male [designated as embryonic day 0 (E0) for the purpose of staging the embryos]. On the required gestational day, pregnant dams were anesthetized with Nembutal $(0.07 \mathrm{mg} / \mathrm{gm}$ body weight; Henry Schein, Melville, NY) until they attained a deep state of anesthesia. The mother's abdominal cavity was opened, and the embryos were removed and perfused with $0.9 \%$ saline, followed by $4 \%$ paraformaldehyde (ICN, Irvine, CA), as described previously (Shu et al., 2000). Once all of the embryos had been removed, the mother was given a lethal dose of Nembutal (0.1 mg/gm).

Coculture assays. E17 embryos were removed from anesthetized dams as described above. Brains were dissected and sectioned coronally on a vibratome. Cortical explants, bisected slices, and glial wedge explants were dissected in L-15 growth medium (Invitrogen, Gaithersburg, MD). Cortical explants or hemisected slices were cocultured with either another cortical explant or a glial wedge explant in collagen gels with DMEM/F-12 medium (Invitrogen), $1 \%$ penicillin-streptomycin, $0.28 \%$ glucose, $1 \%$ of $200 \mathrm{~mm}$ glutamine, $5 \%$ rat serum, and $10 \%$ FBS (Invitrogen, San Diego, CA), as described previously (Shu et al., 2001). After $3 \mathrm{~d}$ in culture, explants were fixed overnight with $4 \%$ paraformaldehyde, and 10\% DiI was injected into the explants to label axon outgrowth. Sytox Green (Molecular Probes, Eugene, OR) was used as a counterstain. Cultures were analyzed by confocal microscopy (Fluoview; Olympus Immunochemicals, Lake Success, NY). The number and length of axons on the proximal and other sides of the explants were measured and compared using two-way ANOVA, followed by post hoc analysis with Newman-Keuls test and paired $t$ tests.

Synthesis of Robol and Robo2 ectodomain proteins. In some experiments, Robo1 and Robo2 ectodomain proteins were added to the culture medium. An in vitro transcription and translation system (TNT T7-coupled reticulocyte lysate system, 14611; Promega, Madison, WI) was used to directly produce the proteins from Robo DNA constructs. The constructs were made by inserting a sequence of the Robol or Robo2 ectodomain fused with a human Fc fragment into the PsectagB plasmid (a gift from Dr. M. Tessier-Lavigne, Stanford University, Stanford, CA), as described previously (Stein and Tessier-Lavigne, 2001).

Immunohistochemistry and in situ hybridization. For Robol/2 and 3A10 immunocytochemistry, $5 \mu \mathrm{m}$ paraffin sections were dewaxed, then treated with $0.3 \%$ hydrogen peroxidase in distilled water for 5 min. After blocking in 2\% BSA in TBS for $10 \mathrm{~min}$ at room temperature, antibodies Robol/2 (see below) or 3A10 (Developmental Studies Hybridoma Bank, Iowa City, IA) (Serafini et al., 1996) were applied overnight at dilutions of either 1:2000 or 1:20 of neat supernatant. Slides were washed in TBS and exposed to a biotinylated swine anti-rabbit and goat 
anti-mouse antibody (1:200 dilution; Dako, Carpinteria, CA), respectively, for $1 \mathrm{hr}$ at room temperature. Slides were then washed in TBS and incubated with the streptavidin, biotin, and peroxidase complex (Strept ABC complex/ HRP; 1:100 dilution; Dako) for $1 \mathrm{hr}$ at room temperature. After three washes for $5 \mathrm{~min}$, slides were incubated with a Tyramide solution (1:150 dilution; NEN, Boston, MA) for $5 \mathrm{~min}$ at room temperature and then washed three times before incubating with the $\mathrm{ABC}$ complex for another $30 \mathrm{~min}$. The chromogenic substrate of peroxidase was a solution of $0.05 \% 3,3^{\prime}$ diaminobenzidine tetrahydrochloride and $0.03 \% \mathrm{H}_{2} \mathrm{O}_{2}$ in $0.05 \mathrm{~mol} / \mathrm{l} \mathrm{TBS}, \mathrm{pH}$ 7.5. The slides were counterstained with Harris' hematoxylin. Normal rabbit preimmune serum was used as a negative control. Positive immunoreactivity was also competed out by the peptide immunogen.

Robol/2 antibody. The Robol/2 rabbit polyclonal antibody was raised against a synthetic peptide corresponding to a 19 amino acid sequence located in the first Ig domain of Robol (RLRQEDFPPRIVEHPSDLI) [Quality Controlled Biochemicals (QBC), Hopkinton, MA] (Hivert et al., 2002). The antibody was affinity purified (QCB) and stored as a stock solution at $1 \mu \mathrm{g} / \mu \mathrm{l}$.

For GFAP immunohistochemistry, $50 \mu \mathrm{m}$ vibratome sections were labeled free-floating. Sections were blocked in a solution consisting of $2 \%(\mathrm{v} / \mathrm{v})$ donkey serum in PBS for $2 \mathrm{hr}$ and then washed in PBS. A rabbit anti-GFAP antibody (1:1000; Dako) was placed on the sections overnight to label the glial wedge. In different experiments, either Cy2- or Cy5-conjugated donkey anti-rabbit secondary antibodies (1:500; Jackson ImmunoResearch Laboratories, West Grove, PA) were used. Cy2 labeling was visualized as green, and Cy5 labeling was visualized as blue by confocal microscopy.

Slit2 in situ hybridization was performed on $10 \mu \mathrm{m}$ cryostat sections as described previously (Shu et al., 2001).

In utero injection of oligonucleotides. Slit 2 antisense oligonucleotides with the sequence $5^{\prime}$-CAGCCAATGCCACTCATCTT- $3^{\prime}$ and a control sequence of 5' -CCGCCACTTCATACATCATT-3' were produced commercially (Oligos, Etc., Ridgefield, CT). Antisense oligonucleotides were designed with the following criteria: (1) to span the translation start codon to inhibit the translation of SLIT2 protein; (2) to contain a roughly equivalent percentage of AT versus GC residues, critical for maintaining a stable structure; (3) not to contain any stable secondary structures, and not to form homodimers; and (4) to ensure that the antisense oligonucleotide was specific for Slit2, a BLAST search of GenBank of the sequence was performed. A sense triplet of the start codon was synthesized within the control oligonucleotide, and a BLAST search of GenBank was also performed on the control oligonucleotide to ensure that it did not bind other known genes in the database. Oligonucleotides contained limited phosphothioate linkages to increase resistance to nuclease degradation ( $\mathrm{Sz}-$ klarczyk and Kaczmarek, 1995). Some oligonucleotides were tagged with fluorescein at both $3^{\prime}$ and $5^{\prime}$ ends to allow the detection of the oligonucleotides after the injection. The oligonucleotides were reconstituted in sterilized water at a concentration of $2 \mu \mathrm{g} / \mu \mathrm{l}$ and stored at $-70^{\circ} \mathrm{C}$. A dosage of $0.5 \mu \mathrm{l}$ of each of the oligonucleotides was injected into the lateral ventricle of each embryo. Timed pregnant mice at either E15 or E16 were used for the in utero injections. Mice were anesthetized with sodium pentobarbital (Nembutal) at $0.07 \mathrm{mg} / \mathrm{gm}$ body weight (diluted in $50 \% \mathrm{MgSO}_{4}$ in saline) and then placed on a warming pad to maintain their body temperature. Once deeply anesthetized, the mother's abdomen was opened to expose the uterus under a dissection microscope. A pulled glass pipette attached to a Picospritzer (General Valve, Fairfield, NJ) was manually inserted into the lateral ventricle through the uterine wall. In the same dam, embryos in one uterine horn were injected with Slit2 antisense oligonucleotides, and embryos in the other uterine horn were injected with control oligonucleotides. After the injection, the mothers' abdomen was sutured closed, and the animal was allowed to recover. After 1 or 2 d survival, the embryos were perfused (on E17) with $0.9 \%$ saline, followed by $4 \%$ paraformaldehyde. DiI or DiA was injected into the medial cortex of fixed brains to label the precrossing or the postcrossing callosal axons. Brains were stored in $4 \%$ paraformaldehyde in light-proof vials at room temperature for 4 weeks to allow time for the diffusion of the dyes in the fixed brains. Brains were then sectioned on a vibratome into $50-\mu \mathrm{m}$-thick sections and labeled for GFAP immunohistochemistry, as described above. Sections were then analyzed by confocal microscopy (Olympus Immunochemicals). DiI and Cy5 labeling were excited with a krypton laser (568 nm emission), and DiA was excited with an argon laser (488 nm emission). Each fluorophore was imaged by single-laser illumination of the sample using barrier filters (Chroma, San Antonio, TX). Each image was scanned two or three times, once for each fluorophore, and then the images were 



GFAP/Slit2 AS-Fluorescein

Figure 3. Slit2 antisense oligonucleotides are localized in glial wedge cells after in utero injection. Sequences of the Slit2 and control antisense oligonucleotides used are shown in $A$. The binding region of the Slit2 antisense is indicated by the alignment $(A)$. Slit2 antisense oligonucleotides were mixed with a small amount of fast green dye to visualize the injection site. The dye remained within the injected hemisphere ( $B$; arrow indicates the injection site). In this case, the injection was performed at E16 and is visualized after perfusion on E17. To localize the Slit2 antisense oligonucleotides, fluorescein-tagged Slit2 antisense oligonucleotides were injected at E16, and brains were perfused after $24 \mathrm{hr}$ and labeled with GFAP immunohistochemistry. Slit2 antisense oligonucleotides ( $C, D$, green dots) were present in the glial wedge, which was identified by GFAP immunohistochemistry $(C, D$, red processes). Slit2 antisense oligonucleotides were located in the ventricular and subventricular zones of the corticoseptal boundary within the soma of the glial wedge cells ( $D$ is a higher-power view of the region delineated in $C$ ). This distribution resembled the expression pattern of Slit2 $\mathrm{mRNA}$ by in situ hybridization ( $F$ is a higher-power view of the boxed region in $E$ ). $B$ is a dorsal view, and $(-F$ are coronal sections. Scale bar (in $F): B, 6 \mathrm{~mm} ; C, 400 \mu \mathrm{m} ; E, 200 \mu \mathrm{m} ; D, F, 50 \mu \mathrm{m}$.

superimposed in Photoshop (Adobe) using a Macintosh G3 computer.

\section{Results}

Glial wedge-mediated repulsion-suppression of cortical axon growth in vitro can be blocked by the addition of Robol and Robo2 ectodomain proteins

In vitro, glial wedge explants can suppress and repel the growth of cortical axons, and in organotypic slice cultures, reorientation of the glial wedge causes axon misrouting (Shu and Richards, 2001). This glial wedge activity can be mimicked by Slit2-expressing cell blocks in vitro. Therefore, because Slit2 is expressed by glial wedge cells in vivo, these data suggested that the repulsive-suppressive guidance activity of the glial wedge may be caused by Slit 2 .

To further investigate this, we performed function blocking experiments by adding soluble Robo1 and Robo 2 ectodomain proteins to collagen gel cocultures of cortex and glial wedge. Both Robol and Robo 2 bind Slit2 with high affinity, and both are expressed in cortical neurons; therefore, if the activity is caused by Slit2, the addition of Robo1/2 ectodomain proteins should bind to Slit2 expressed by the glial wedge and diminish the repulsivesuppressive activity. Robo1 and Robo2 ectodomain proteins translated in vitro had an expected molecular weight of 150$160 \mathrm{kDa}$ (Fig. 1A). As described previously, in cortex-cortex explant cocultures $(n=11)$, axons grew into the apposing cortical explant (Fig. $1 B$ ). However, when cortex was cultured with glial wedge $(n=$ 11 ), significantly fewer axons grew into the glial wedge explant (Fig. 1C,F; 1.6 vs 15.6 axons; $p<0.01$; post hoc analysis with Newman-Keuls test). Axons growing on the side proximal to the glial wedge were also significantly shorter compared with either axons growing on the other three sides of the explant in the same culture (164.7 $\mu \mathrm{m}$ vs $228.5 \mu \mathrm{m}$; paired $t$ test; $p<$ 0.05 ; Fig. $1 C, F)$ or compared with axons growing in cortex-cortex cocultures on the proximal side (mean length, 285.9 $\mu \mathrm{m}$ ) or the other three sides (mean length, $263.8 \mu \mathrm{m}$; Fig. $1 B, F)$. In cultures in which the Robo1/2 ectodomain proteins were added, the respulsive-suppressive activity of the glial wedge could be blocked in a titratable manner. In these cultures, significantly more axons grew into the glial wedge explant (for $2 \mathrm{ng} / \mu \mathrm{l}$ : 6.4 vs $1.6 \mathrm{ax}$ ons; $p<0.05$; for $20 \mathrm{ng} / \mu \mathrm{l}$ : 10 vs 1.6 axons; $p<0.01$; post hoc analysis with NewmanKeuls test). Furthermore, axons facing the glial wedge grew to control lengths in the presence of either $2 \mathrm{ng} / \mu \mathrm{l}(255.0 \mu \mathrm{m}$ vs $266.1 \mu \mathrm{m})$ or $20 \mathrm{ng} / \mu \mathrm{l}(256.7 \mu \mathrm{m}$ vs 226.4 $\mu \mathrm{m})$ of Robo ectodomain proteins.

These data suggest that in vitro the repulsive-suppressive activity (or a major component of it) expressed by the glial wedge binds to Robo ectodomain proteins and is, therefore, likely to be Slit2.

\section{Callosal axons express Robo receptors}

We previously showed that both Robo1 and Robo2 were expressed in the cortical plate at E17, a time when the corpus callosum is forming (Shu and Richards, 2001). However, because this experiment was performed by in situ hybridization, we could not be sure that these receptors were expressed on callosal axons. Using a pan Robo1 and Robo2 antibody, we find that callosal 
axons express these receptors at high levels at E15-E17 (Fig. 2), a time when callosal axons are actively pathfinding. Robo antibodies labeled callosal axons as they approached the midline at E15 (Fig. $2 A, B$, arrow) and crossed at E16 (Fig. 2C,D, arrow). At E17, Robo immunostaining of the corpus callosum overlapped with 3A10 immunolabeling, which labels neurofilaments (Fig. 2E, F, arrow). The lateral cortical projection through the internal capsule was also labeled by the Robo antibody (Fig. 2C, arrow). This expression, along with phenotypes observed in the Slit2 knockout mouse (Bagri et al., 2002), suggests that in vivo callosal and laterally projecting cortical axons are guided by Slit 2 through Robo receptors.

\section{Slit2 antisense oligonucleotides bind to regions of Slit2 expression in vivo}

To investigate the role of Slit2 in guiding callosal axons at the midline in vivo, we generated Slit2 antisense oligonucleotides and injected them into the lateral ventricle of mouse embryonic brains in utero. Slit2 antisense and control oligonucleotides (Fig. $3 A$ ) were injected into brains using a pulled glass micropipette attached to a picospritzer. A small amount of fast green dye, used for visualization during the injection procedure, indicated that injections were largely confined to a single cortical hemisphere (Fig. 3B, arrow). Oligonucleotides were end-labeled with fluorescein, so that they could be visualized after sectioning to determine their distribution. In all cases analyzed $(n=6)$, Slit2 oligonucleotides localized to the corticoseptal boundary (Fig. $3 C, D$ ), a region of high Slit2 expression (Fig. $3 E, F$ ). Colabeling with an anti-GFAP antibody (Fig. 3C,D, red labeling) indicated that Slit2 oligonucleotides (Fig. 3C,D, green labeling) were present in glial wedge cells. Control oligonucleotides were also present in the glial wedge but tended to be more widely distributed at the corticoseptal boundary (see Fig. $5 F$ ). One possible explanation for this wider distribution is that the control oligonucleotides did not bind to specific mRNAs within the cells. Therefore, Slit2 oligonucleotides preferentially bind to regions of Slit2 expression, enabling them to cause a downregulation of Slit2 protein expression, as has been described previously for this technique (Toulme, 1997). Labeled oligonucleotides were only observed in brains after $24 \mathrm{hr}$ and were no longer observed at $48 \mathrm{hr}$. This probably reflects a degradation of the oligonucleotides by $48 \mathrm{hr}$, making this technique very precise for regulating protein expression on a single developmental day.

\section{Slit2 antisense oligonucleotides cause aberrant callosal axon pathfinding of precrossing axons}

Because the first callosal axons cross the midline at E15.5 (Rash and Richards, 2001), Slit2 antisense oligonucleotides were injected into a single lateral ventricle of the brains of either E15 (Fig. 4) or E16 (Fig. 5) mouse pups in utero. All embryos were then perfused at E17, injected with DiI, and later processed for GFAP immunohistochemistry. In brains injected with control antisense oligonucleotides ( $n=6$ at E15 and $n=11$ at E16), callosal axons crossed the midline and grew dorsally into the opposite hemisphere (Figs. 4A, $C, 5 A, B, F$, red axons). In Slit2 antisense oligonucleotide-injected brains at E15 ( $n=5$ of 6 ), large numbers of callosal axons either deviated from the main bundle (Fig. $4 D$ ) or failed to cross the midline completely, beginning to form Probst-like bundles (Fig. $4 \mathrm{~B}$, asterisk) or growing aberrantly into the septum (Fig. $4 \mathrm{~B}$, arrowhead; Table 1). Even after only a $24 \mathrm{hr}$ survival period, we observed dramatic errors in callosal axon pathfinding in the presence of the Slit2 antisense oligonucleotides ( $n=12$ of 13 displayed pathfinding errors). The phenotypes


Figure 4. In utero injection of Slit2 antisense oligonucleotides disrupts precrossing callosal axon pathfinding at midline on $\mathrm{E} 15$. Control $(A, C)$ or Slit2 antisense $(B, D)$ oligonucleotides were injected into the right lateral ventricle of E15 mouse embryos. On E17, the injected embryos were perfused, and after postfixation, Dil was injected into the medial cortex to label callosal axons (axons labeled red in all panels). GFAP immunohistochemistry was used to label the glial wedge (GW) ( $A$, $B$, green labeling; $C, D$, pseudo-blue labeling). Control oligonucleotide-injected brains developed a normal corpus callosum (CC) ( $A$, $C$; two different brains). Brains injected with Slit2 antisense oligonucleotides displayed dramatic axon pathfinding errors at the midline. Many callosal axons stalled at the midline and failed to cross, sometimes forming Probst bundle-like structures ( $B$, asterisk). Some axons projected aberrantly into the septum ( $B$, arrow). Unlabeled oligonucleotides were used in $A$ and $B$, and fluorescein-labeled oligonucleotides were used in $C$ and $D$; however, the fluorescein tag could not be detected after the $2 \mathrm{~d}$ survival period $(C, D)$. All sections are in the coronal plane. Scale bar (in $D): A, 300 \mu \mathrm{m} ; B, 250$ $\mu \mathrm{m} ; C, D, 150 \mu \mathrm{m}$.

varied from subtle deviations from the main bundle (Fig. 5C) to more severe phenotypes, where the majority of axons failed to cross the midline and grew into the septum (Fig. 5D,E; Table 1). In brains injected with FITC end-labeled oligonucleotides, both Slit2 and control antisense oligonucleotides were present at the corticoseptal boundary (Fig. $5 F, G$, green dots), but only brains injected with Slit2 antisense oligonucleotides displayed axonal pathfinding errors (Fig. 5G) compared with controls (Fig. 5F, Table 1). The more severe cases resembled the phenotype observed in Slit2 knock-out mice (Bagri et al., 2002), indicating that the variability observed was probably because of variations in the amount of Slit protein "knockdown" achieved between brains.

\section{Postcrossing callosal axons are guided by Slit 2 and the glial wedge}

The glial wedge is a bilaterally symmetrical structure, present on either side of the midline at the corticoseptal boundary (Shu and Richards, 2001). To investigate the role of Slit2 and the glial wedge in regulating the growth trajectories of callosal axons after they cross the midline, we injected control or Slit2 antisense oligonucleotides into brains and then simultaneously analyzed both precrossing and postcrossing axons. Oligonucleotides were injected on E16, and brains were perfused on E17 and injected with DiA in the same hemisphere to label precrossing axons and with DiI in the opposite hemisphere to label postcrossing axons (Fig. 6A).

In control-injected brains $(n=8)$, callosal axons from both hemispheres crossed the midline and overlapped within the tract of the corpus callosum (Fig. $6 B, C$ ). No axons from either hemisphere were observed deviating from the main bundle. In Slit2 antisense oligonucleotide-injected brains, precrossing callosal axons were again observed deviating from the main bundle and 

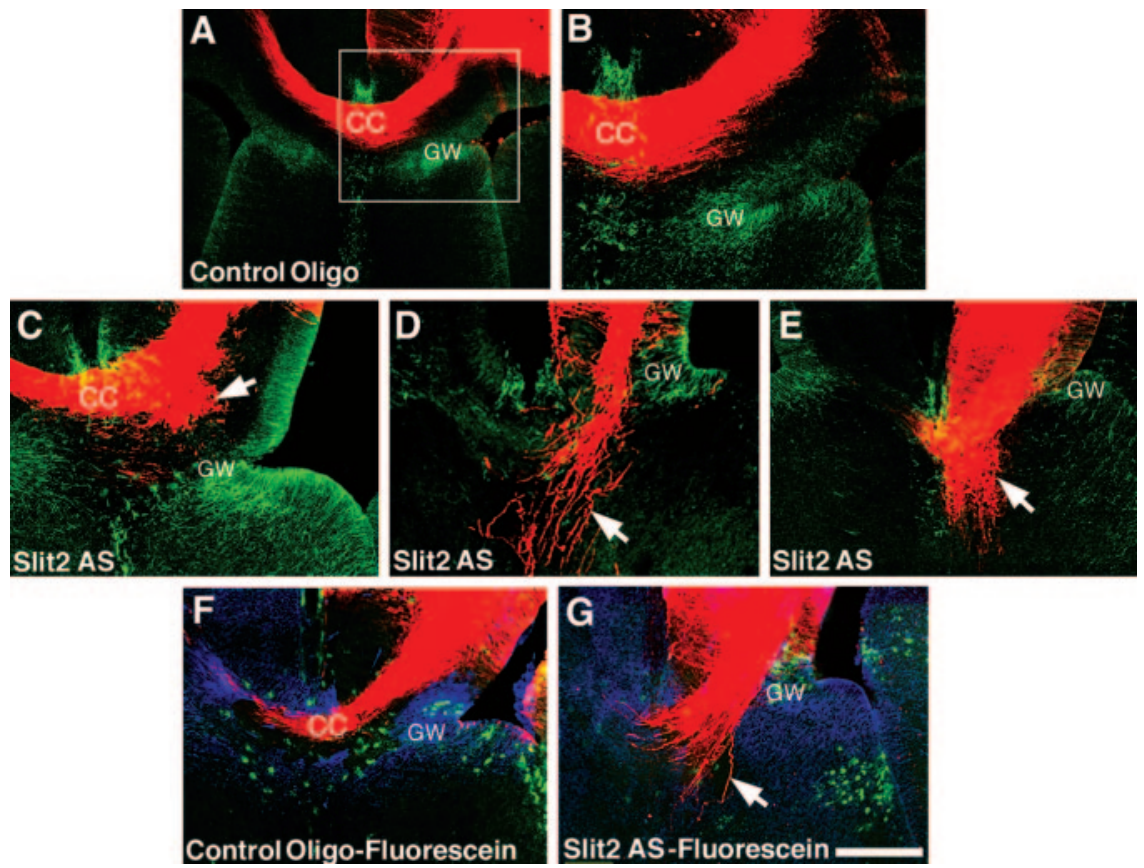

Figure 5. Pathfinding errors of callosal axons in brains injected with Slit2 antisense oligonucleotides at E16. E16 brains injected with control oligonucleotides displayed normal callosal pathfinding $(A, B, F$, Dil-labeled red axons; $B$ is a higher-power view of the region delineated in $A$ ). However, brains injected with Slit2 antisense oligonucleotides displayed varying degrees of pathfinding errors ( $C-E, G$, Dil-labeled red axons). In some brains, axons deviated from the main bundle $(C$, arrow), and in others the majority of axons grew aberrantly into the septum ( $D, E, G$, arrows). Some experiments were performed with fluorescein-tagged control $(F)$ or Slit2 antisense $(G)$ oligonucleotides and could be detected at the cortiocseptal boundary and within the glial wedge $(F, G$, green dots). All sections are in the coronal plane and represent different brains in each panel, except in $A$ and $B$. The glial wedge is labeled with GFAP immunohistochemistry in green in $A-E$ and in pseudo-blue in Fand $G$. Scale bar (in G): $A, 400 \mu \mathrm{m} ; B, D, 150 \mu \mathrm{m} ; C, E-G$, $200 \mu \mathrm{m}$.

Table 1. Pathfinding defects observed in oligonucleotide-injected brains

\begin{tabular}{lllll}
\hline & $\begin{array}{l}\text { No obvious } \\
\text { pathfinding } \\
\text { defects }\end{array}$ & $\begin{array}{l}\text { Subtle } \\
\text { pathfinding } \\
\text { defect }\end{array}$ & $\begin{array}{l}\text { Mild } \\
\text { pathfinding } \\
\text { defect }\end{array}$ & $\begin{array}{l}\text { Severe } \\
\text { pathfinding } \\
\text { defect }\end{array}$ \\
\hline $\begin{array}{c}\text { Precrossing axons } \\
\text { E15 antisense }\end{array}$ & 1 & 1 & 2 & 2 \\
E15 control & 6 & 0 & 0 & 0 \\
E16 antisense ${ }^{a}$ & 3 & 2 & 8 & 9 \\
E16 control & 19 & 0 & 0 & 0 \\
Postcrossing axons & & 2 & & 1 \\
E16 antisense ${ }^{a}$ & 2 & 0 & 4 & 0 \\
E16 control $^{\text {Est }}$ & 8 & 2 & 0 &
\end{tabular}

Precrossing axons were defined as axons labeled with a carbocyanine dye injection in the same hemisphere as the oliognucleotide injection. These were pooled from brains labeled in with a dye injection in only one hemisphere and brains labeled with a dye injection in both hemispheres. Postcrossing axons were analyzed from brains injected with a dye in both hemispheres. Pathfinding defects were defined as "subtle" if the axons were only defasiculated, "mild" in cases in which the axons were both defasiculated and a few axons grew aberrantly into the septum or the glia wedge, and "severe" in cases in which the axons were defasiculated and most grew into the septum or the glial wedge or formed Probst-like bundles.

${ }^{a}$ Note that in the two cases of antisense oligonucleotide-injected brains in which postcrossing axons displayed no obvious pathfinding defects, the precrossing axons in the same brain were also unaffected and make up two-thirds of the brains indicated as having no obvious pathfinding defects in the precrossing total. In these cases, it is, therefore, possible that the antisense oliognucleotide injections were not effective in reducing the levels of Slit2 protein.

growing into the septum (Fig. $6 D-H$, green axons, $E, F, H$, arrowheads; $n=7$ of 9 displayed pathfinding errors; Table 1). In this experiment, axons from the contralateral hemisphere that had already encountered endogenous levels of Slit2 protein expressed in the untreated hemisphere encountered presumably lower levels of Slit 2 protein once they crossed the midline. In $100 \%$ of cases in which the precrossing axons displayed pathfinding errors $(n=$ 7 of 7; Table 1), postcrossing axons were also observed growing aberrantly into the contralateral septum (Fig. 6E, F, red axons labeled with arrows) or deviating from the main bundle (Fig. $6 G, H$, arrows). In more severe cases, some axons from the contralateral hemisphere did not cross the midline and began to form Probst-like bundles (Fig. $6 \mathrm{H}$, red axons labeled by an asterisk).

These results indicated that Slit2 expressed by the glial wedge was required for maintaining axons within the main callosal bundle and guiding them dorsally into the contralateral hemisphere and away from the midline. However, given the high degree of fasciculation and intermingling of axons from each hemisphere that occurs within the callosal bundle (Fig. $6 B, C)$, the possibility existed that postcrossing axons were effected by the aberrant growth trajectories of precrossing axons and not directly by Slit 2 and the glial wedge. To investigate this, we devised a novel hemisected slice preparation to specifically observe the growth of postcrossing callosal axons (Fig. 7A). Slices of living E17 brains were bisected at the midline and then cultured in collagen gels. After fixation, DiI was injected into the callosal pathway to label postcrossing axons that grew out of the slice and into the collagen gel that was covering the slice. Postcrossing callosal axons could be specifically analyzed in this assay and grew abundantly into the collagen when cultured alone $(n=7$; Fig. $6 B, C)$. When explants of neocortex were placed in the path of these axons $(n=$ 7 ), postcrossing callosal axons grew into the cortical explants (Fig. 6D,E). However, when explants of glial wedge were placed in the pathway $(n=10)$, fewer axons grew out of the hemisected slice (7 vs 20 axons, respectively; post hoc analysis with NewmanKeuls test; $p<0.01$; Fig. $7 K)$, and very few entered the glial wedge ( 5 vs 0.7 axons, respectively; $p<0.01$; Figs. $6 F, G, 7 J$ ). To examine whether this suppression-repulsion was caused, at least in part, by the expression of Slit2, we added $20 \mathrm{ng} / \mu \mathrm{l}$ Robol and Robo2 ectodomain proteins to the glial wedge-hemisected cortex cultures. In these cultures $(n=7)$, significantly more postcrossing callosal axons were now able to grow into the glial wedge explants ( 7 vs 0.7 axons, respectively; $p<0.001$ ) and were longer in the presence of the Robol/2 ectodomain proteins $(210 \mu \mathrm{m}$ vs $110 \mu \mathrm{m} ; p<0.001$; Fig. $7 L)$. Hence, the addition of Robol and Robo2 ectodomain proteins was able to block the suppressiverepulsive effect of the glial wedge.

These data suggest that Slit2 and the glial wedge are required for the correct axonal pathfinding of callosal axons on both sides of the midline.

\section{Discussion}

Previous studies have suggested that Slit 2 is required for precrossing callosal axons to turn medially and cross the midline (Shu and Richards, 2001; Bagri et al., 2002). This repulsion effectively inhibits callosal axons from entering the septum and causes them to turn medially. What was unclear was whether callosal axons were guided by the contralateral glial wedge after they crossed the midline. In the mammalian spinal cord, commissural axons become 
responsive to Slit only after they have crossed the midline (Zou et al., 2000). Slit, therefore, inhibits these axons from recrossing the midline once they have crossed. The small size of the developing spinal cord and the ventral location of the commissure make it difficult to ectopically misexpress genes in this system in vivo without performing genomic manipulations. Thus, the developing corpus callosum provides a more accessible system to analyze gene function and commissure formation in vivo in mammals through the use of techniques such as antisense oligonucleotides, in utero injection of viral vectors, or in utero electroporation. Furthermore, data provided here and elsewhere (Erskine et al., 2000; Shu and Richards, 2001; Bagri et al., 2002; Hutson and Chien, 2002; Plump et al., 2002) suggest that the function of Slit2 at the midline of the brain may be different from that of the spinal cord. In the brain, axons respond to Slit both before crossing the midline as well as after they cross. One mechanism by which this may occur is through the direct interaction of the Netrin-1 receptor deleted in colorectal carcinoma (DCC) with Robo1/2. Recent evidence suggests that the cytoplasmic domains of Robol and DCC can interact (Stein and Tessier-Lavigne, 2001) and that both receptors may operate together to mediate repulsion in an UNC-6-Netrin-1independent manner (Yu et al., 2002). Becausecallosal axons express both Robo1/2 and DCC (Shu et al., 2000), these receptors may operate together within this system to mediate repulsion on both sides of the midline.

In vivo, postcrossing callosal axons displayed varying morphologies in the Slit2 antisense oligonucleotideinjected brains. Some axons deviated from the main bundle and grew down into the septum, whereas others formed bundles of axons at the midline and did not enter the contralateral hemisphere. These data suggest that in addition to keeping callosal axons out of the septum as they cross the midline, Slit2 may be required for axons to cross the midline and enter the contralateral hemisphere. Given the mistargeting of axons on the same side as the antisense oligonucleotide injections, it was reasonable to assume that the mistargeting of contralateral axons may be caused by the fasciculation of these axons with the aberrantly targeting ipsilateral axons. However, in vitro analysis of postcrossing callosal axons independent of projections from the contralateral hemisphere suggests that they still respond to the glial wedge even after they cross the midline. This evidence does not exclude the possibility that some of the postcrossing axons were led astray by fasciculating with mistargeting axons from the ipsilateral hemisphere, however, we did not observe this in our experiments.

Distinct differences exist in the way Slit2 regulates commissural axon growth in the mammalian spinal cord and brain

In the mammalian spinal cord, Slit2 acts on commissural axons only after they have crossed the midline (Zou et al., 2000), pre-
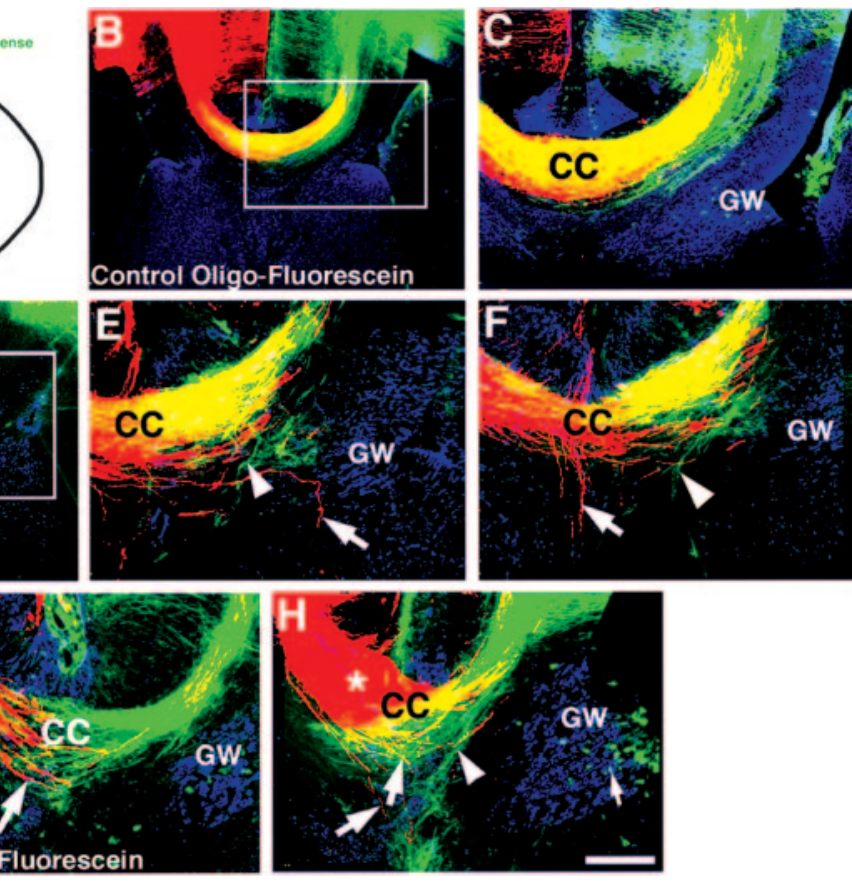

Figure 6. Disruption of postcrossing callosal axons in vivo by Slit2 antisense oligonucleotides. To investigate the role of Slit2 in regulating the growth of postcrossing callosal axons, we injected either control oligonucleotides $(B, C)$ or Slit2 antisense oligonu(D-H) into the right lateral ventricle of E16 embryos in utero. Embryos were perfused on E17 and later labeled with either ed Probst-like bundles ( $H$, red axons and bundle labeled by an asterisk; $G$ and $H$ are examples from two additional brains). All sections are in the coronal plane. Fluorescein-labeled oligonucleotides were used in $B, C, G$, and $H$ (green dots in these panels; $H$, small arrow); unlabeled oligonucleotides were used in D-F. Scale bar (in $H$ ): B, D, $200 \mu \mathrm{m} ; C, E-H, 100 \mu \mathrm{m}$.

venting them from recrossing the midline as they project rostrally just lateral to the floorplate. In the developing visual system, Slit1 and Slit 2 act in concert on precrossing retinal ganglion cell (RGC) axons, channeling them into the optic tract as they grow toward the chiasm (Plump et al., 2002). In Slit1/2 double knock-out mice, many RGC axons cross aberrantly in ectopic commissures anterior to the optic chiasm (Plump et al., 2002). Furthermore, unlike the Drosophila nerve cord, Slit molecules do not act to regulate ipsilateral versus contralateral axon crossing in the mammalian visual system (Erskine et al., 2000; Plump et al., 2002). These data suggest that in the ventral forebrain Slits act by channeling the axons within the tract and specifying where they cross the midline. As in the visual system, Slit2 expressed by the glial wedge may regulate the position of the midline callosal crossing point (in the dorsal-ventral axis rather than the anterior-posterior axis). Given this model and assuming that midline fusion had occurred more dorsally, it is possible that ectopic expression of Slit 2 more dorsally could force callosal axons to cross more dorsally before their reaching the cortiocseptal boundary. However, it is highly likely that additional molecules expressed within this region act together with Slit2 to regulate callosal axon guidance. Evidence for this is that, in Slit2 knockout mice, callosal axons initially penetrate the septum but then form classic Probst bundles at the corticospetal boundary rather 
A

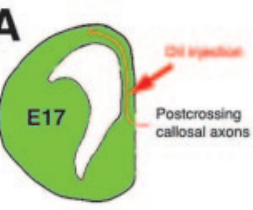

hemisected brain slice
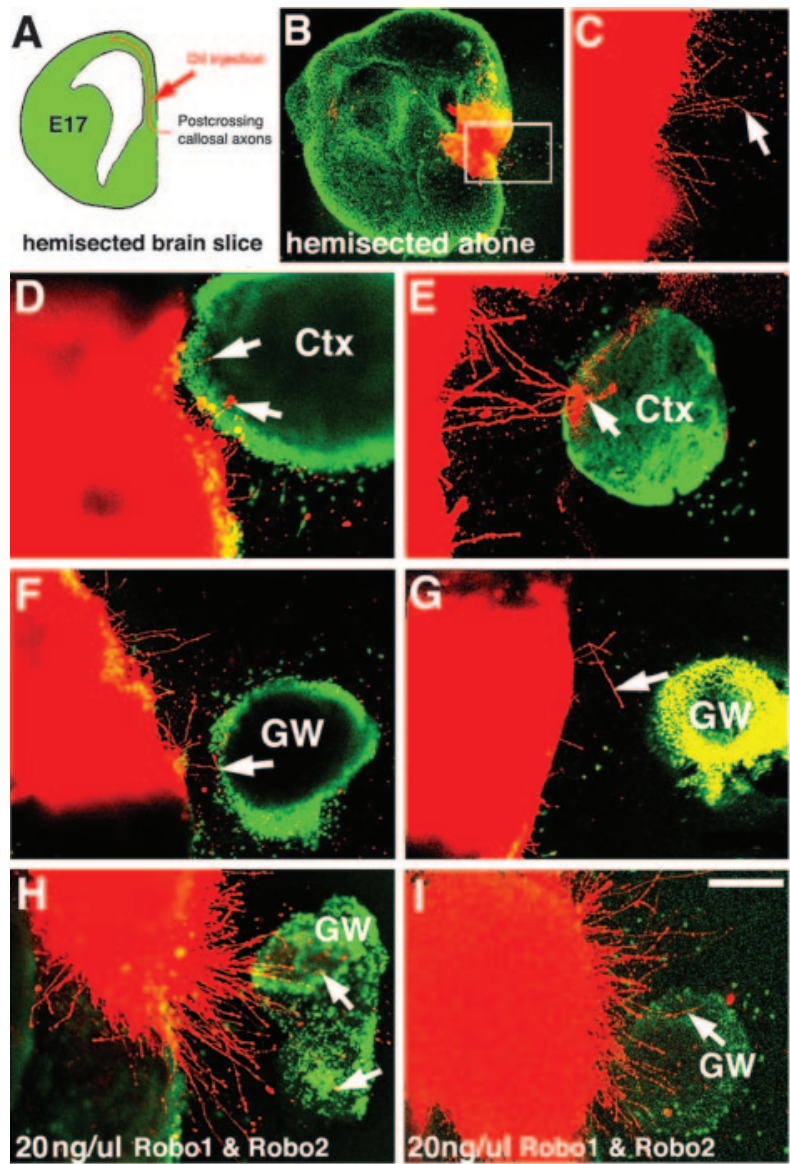

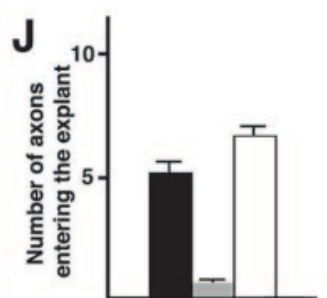

K



$\mathbf{L}$

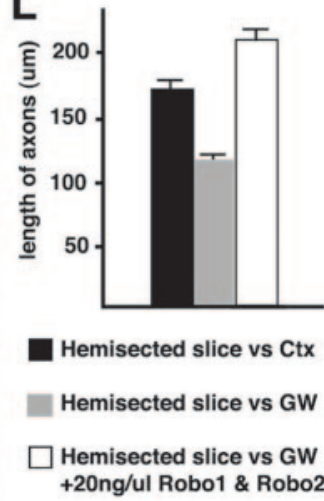

Figure 7. The glial wedge supresses-repels the growth of postcrossing callosal axons in vitro. To specifically study postcrossing callosal axons, we made live coronal slices of E17 brains and bisected them along the midline to obtain slices containing only one hemisphere. These hemisected slices were grown in collagen for $3 \mathrm{~d}$ and then fixed and labeled with the cellular marker Sytox green (green cellular labeling in all panels). Callosal axons were visualized with Dil injected into the medial cortex of the slice $(A-I$, red axons). A schematic of the slice culture is shown in $A$. When slices were cultured alone, callosal axons crossed the midline and grew into the collagen $(B, C$, arrow; $C$ is a higher-power view of the region delineated in $B)$. Hemisected slices cocultured with cortical explants ( $D, E$; two different examples) had callosal axons that grew into the collagen and entered the cortical explants ( $D$, $E$, arrows). However, when hemisected slices were cocultured with glial wedge explants, fewer callosal axons left the slice and entered the glial wedge ( $F, G$; two different examples). In many cases, as the callosal axons approached the glial wedge, they changed direction and turned away $(F, G$, arrows). To investigate whether this repulsive-suppressive effect on the growth of postcrossing callosal axons was caused by Slit2, we added $20 \mathrm{ng} / \mu$ l Robo1 and Robo2 ectodomain proteins to the cultures. Two examples of these cultures are shown in $H$ and $I$, where callosal axons were now able to grow into the glial wedge explant $(H, I$, arrows). Quantitation of these effects is shown in $J-L$; the mean and SEM are shown. The number of axons entering the explant is shown in J. The number and the length of axons that exited the hemisected slice (regardless of whether they entered the opposing slice) are shown in $K$ and $L$, respectively. ${ }^{*} p<0.01$ (in J, K); ${ }^{*} p<0.001$ (in L). GW, Glial wedge explant; Ctx, cortex explant. Scale bar (in I): $B, 400 \mu \mathrm{m} ;(-l, 150 \mu \mathrm{m}$.

than forming them more ventrally. Thus, additional molecules must be expressed that prevent axons from remaining within the septal region.

Why is there a difference in Slit2 guidance between the brain and spinal cord? It is probably because of morphological differences between the structures present at the midline and, consequently, the expression of Slit2 at the midline. In the Drosophila nerve cord and the mammalian spinal cord, midline glia and the floorplate respectively, provide a single source of Slit2 expression, whereas the bilateral glial wedges of the cortical midline provide two sources of Slit2. Furthermore, in the Drosophila nerve cord and the mammalian spinal cord, axons remain in close proximity to the midline after they cross and project rostrally or caudally. In the brain, callosal axons leave the midline, growing away to enter the contralateral hemisphere.
Slit molecules play a role in axon fasciculation in the mammalian brain In addition to their roles in axon repulsion, Slit1 and Slit2 have been implicated in regulating axon fasciculation in the visual system (Ringstedt et al., 2000; Plump et al., 2002). In Slit $1 / 2$ knock-out mice, RGC axons are defasciculated within the optic tract and chiasm (Plump et al., 2002). Here, we also saw axon defasciculation occurring within the callosal tract in Slit2 oligonucleotide-injected brains. Possible explanations for this are that the defasciculation observed is a form of mild repulsion that normally acts to "channel" the axons within the tract, or that within the tract itself Slit2 promotes axon fasciculation through its interaction with molecules of the extracellular matrix such as laminin (Nguyen-Ba-Charvet et al., 2001) and heparan sulfate proteoglycans $(\mathrm{Hu}$, 2001). Alternatively, Slit may act differentially within the same commissural tract by repelling some axons and causing the fasiculation of other axons. This could be mediated through a variation in the concentration of Slit2 presented to different axons (e.g., those on the outside might encounter a higher concentration than those on the inside of the bundle) or by the expression of different Robo receptors on the axons. In Drosophila, the expression of various combinations of Robo receptors specifies whether longitudinally projecting axons will be strongly or more weakly repulsed by a gradient of Slit (Rajagopalan et al., 2000; Simpson et al., 2000; Goodhill, 2003). There is currently no evidence that the Robo receptors are differentially expressed on axons within the callosal tract or that different axons within the tract respond differently to Slit. Reagents necessary to study the protein distribution of Slit and the Robo receptors are currently not available.

Thus, although there are many similarities in the way in which Slit and Robo molecules function during development across species, even within the same species, major differences in function exist, as described here, between the mammalian spinal cord and brain.

\section{References}

Bagri A, Marin O, Plump AS, Mak J, Pleasure SJ, Rubenstein JLR, TessierLavigne M (2002) Slit proteins prevent midline crossing and determine the dorsoventral position of major axonal pathways in the mammalian forebrain. Neuron 33:233-248.

Brose K, Bland KS, Wang KH, Arnott D, Henzel W, Goodman CS, TessierLavigne M, Kidd T (1999) Slit proteins bind Robo receptors and have an evolutionary conserved role in repulsive axon guidance. Cell 96:795-806.

Cummings DM, Malun D, Brunjes PC (1997) Development of the anterior commissure in the opossum: midline extracellular space and glia coincide with early axon decussation. J Neurobiol 32:403-414.

Erskine L, Williams SE, Brose K, Kidd T, Rachel RA, Goodman CS, TessierLavigne M, Mason CA (2000) Retinal ganglion cell axon guidance in the 
mouse optic chiasm: expression and function of Robos and slits. J Neurosci 20:4975-4982.

Goodhill GJ (2003) A theoretical model of axon guidance by the Robo code. Neural Comput 15:549-564.

Hivert B, Liu Z, Chuang CY, Doherty P, Sundaresan V (2002) Robol and robo2 are homophilic binding molecules that promote axonal growth. Mol Cell Neurosci 21:534-545.

Hu H (1999) Chemorepulsion of neuronal migration by Slit2 in the developing mammalian forebrain. Neuron 23:703-711.

Hutson LD, Chien CB (2002) Pathfinding and error correction by retinal axons: the role of astray/robo2. Neuron 33:205-217.

Jacobs JR, Goodman CS (1989) Embryonic development of axon pathways in the Drosophila CNS. I. A glial scaffold appears before the first growth cones. J Neurosci 9:2402-2411.

Kidd T, Brose K, Mitchell KJ, Fetter RD, Tessier-Lavigne M, Goodman CS, Tear G (1998) Roundabout controls axon crossing of the CNS midline and defines a novel subfamily of evolutionarily conserved guidance receptors. Cell 92:205-215.

Kidd T, Bland KS, Goodman CS (1999) Slit is the midline repellent for the Robo receptor in Drosophila. Cell 96:785-794.

Li HS, Chen JH, Wu W, Fagaly T, Zhou L, Yuan W, Dupuis S, Jiang ZH, Nash W, Gick C, Ornitz DM, Wu JY, Rao Y (1999) Vertebrate slit, a secreted ligand for the transmembrane protein roundabout, is a repellent for olfactory bulb axons. Cell 96:807-818.

Marcus RC, Blazeski R, Godement P, Mason CA (1995) Retinal axon divergence in the optic chiasm: uncrossed axons diverge from crossed axons within a midline glial specialization. J Neurosci 15:3716-3729.

Mori K, Ikeda J, Hayaishi O (1990) Monoclonal antibody R2D5 reveals midsaggittal radial glial system in postnatally developing and adult brainstem. Proc Natl Acad Sci USA 87:5489-5493.

Nguyen-Ba-Charvet KTN, Brose K, Marillat V, Kidd T, Goodman CS, Tessier-Lavigne M, Sotelo C, Chedotal A (1999) Slit2-Mediated chemorepulsion and collapse of developing forebrain axons. Neuron 22:463-473.

Nguyen-Ba-Charvet KT, Brose K, Marillat V, Sotelo C, Tessier-Lavigne M, Chedotal A (2001) Sensory axon response to substrate-bound Slit2 is modulated by laminin and cyclic GMP. Mol Cell Neurosci 17:1048-1058.

Pires-Neto M, Braga-De-Souza S, Lent R (1998) Molecular tunnels and boundaries for growing axons in the anterior commissure of hamster embryos. J Comp Neurol 399:176-188.

Plump AS, Erskine L, Sabatier C, Brose K, Epstein CJ, Goodman CS, Mason CA, Tessier-Lavigne M (2002) Slit1 and Slit2 cooperate to prevent premature midline crossing of retinal axons in the mouse visual system. Neuron 33:219-232.

Polleux F, Giger RJ, Ginty DD, Kolodkin AL, Ghosh A (1998) Patterning of cortical efferent projections by semaphorin-neuropilin interactions. Science 282:1904-1906.

Rajagopalan S, Vivancos V, Nicolas E, Dickson BJ (2000) Selecting a longitudinal pathway: Robo receptors specify the lateral position of axons in the Drosophila CNS. Cell 103:1033-1045.

Rash BG, Richards LJ (2001) A role for cingulate pioneering axons in the development of the corpus callosum. J Comp Neurol 434:147-157.

Richards LJ (2002) Axonal pathfinding mechanisms at the cortical midline and in the development of the corpus callosum. Braz J Med Biol Res 35:1431-1439.
Ringstedt T, Braisted JE, Brose K, Kidd T, Goodman C, Tessier-Lavigne M, O'Leary DDM (2000) Slit inhibition of retinal axon growth and its role in retinal axon pathfinding and innervation patterns in the diencephalon. J Neurosci 20:4983-4991.

Serafini T, Colamaino SA, Leonardo ED, Wang H, Beddington R, Skarnes WC, Tessier-Lavigne M (1996) Netrin-1 is required for commissural axon guidance in the developing vertebrate nervous system. Cell 87:1001-1014.

Shen Y, Mani S, Donovan SL, Schwob JE, Meiri KF (2002) Growthassociated protein-43 is required for commissural axon guidance in the developing vertebrate nervous system. J Neurosci 22:239-247.

Shu T, Richards LJ (2001) Cortical axon guidance by the glial wedge during the development of the corpus callosum. J Neurosci 21:2749-2758.

Shu T, Valentino KM, Seaman C, Cooper HM, Richards LJ (2000) Expression of the netrin-1 receptor, deleted in colorectal cancer (DCC), is largely confined to projecting neurons in the developing forebrain. J Comp Neurol 416:201-212.

Shu T, Shen W-B, Richards LJ (2001) The development of projections within the perforating pathway that intersects the corpus callosum. J Comp Neurol 436:411-422.

Shu T, Butz KG, Plachez C, Gronostajski RM, Richards LJ (2003) Abnormal development of forebrain midline glia and commissural projections in Nfia knock-out mice. J Neurosci 23:203-212.

Simpson JH, Bland KS, Fetter RD, Goodman CS (2000) Short range and long range guidance by Slit and its Robo receptors: a combinatorial code of Robo receptors controls lateral position. Cell 103:1019-1032.

Snow DM, Steindler DA, Silver J (1990) Molecular and cellular characterization of the glial roof plate of the spinal cord and optic tectum: a possible role for a proteoglycan in the development of an axon barrier. Dev Biol 38:359-376.

Stein E, Tessier-Lavigne M (2001) Hierarchical organization of guidance receptors: silencing of netrin attraction by slit through a Robo/DCC receptor complex. Science 291:1928-1938.

Szklarczyk A, Kaczmarek L (1995) Antisense oligodeoxyribonucleotides: stability and distribution after intracerebral injection into rat brain. J Neurosci Methods 60:181-187.

Toulme J (1997) Antisense oligodeoxynucleotides and antisense RNA. In: Novel pharmacological and therapeutic agents (Weiss B, ed). New York: CRC.

Van Hartesveldt C, Moore B, Hartman B (1986) Transient midline raphe glial structure in the developing rat. J Comp Neurol 253:175-184.

Wang KH, Brose K, Arnott D, Kidd T, Goodman CS, Henzel W, TessierLavigne M (1999) Biochemical purification of a mammalian slit protein as a positive regulator of sensory axon elongation and branching. Cell 96:771-784.

Wu DY, Schneider GE, Silver J, Poston M, Jhaveri S (1998) A role for tectal midline glia in the unilateral containment of retinocollicular axons. J Neurosci 18:8344-8355.

Yu TW, Hao JC, Lim W, Tessier-Lavigne M, Bargmann CI (2002) Shared receptors in axon guidance: SAX3/Robo signals via UNC-34/Enabled and a Netrin-independent UNC-40/DCC function. Nat Neurosci 5:1147-1154.

Zou Y, Stoeckli E, Chen H, Tessier-Lavigne M (2000) Squeezing axons out of the gray matter: a role for slit and semaphorin proteins from midline and ventral spinal cord. Cell 102:363-375. 